\title{
Christine Macleod, Heroes of invention. Technology, liberalism and British identity 1750-1914
}

Cambridge, Cambridge university press, 2007, 458 pages.

\section{Liliane Pérez}

\section{OpenEdition}

\section{Journals}

Édition électronique

URL : http://journals.openedition.org/dht/285

DOI : $10.4000 /$ dht.285

ISSN : 1775-4194

Éditeur :

Centre d'histoire des techniques et de l'environnement du Cnam (CDHTE-Cnam), Société des élèves du CDHTE-Cnam

\section{Édition imprimée}

Date de publication : 1 décembre 2009

Pagination : 238-241

ISBN : 978-2-9530779-4-0

ISSN : 0417-8726

\section{Référence électronique}

Liliane Pérez, «Christine Macleod, Heroes of invention. Technology, liberalism and British identity 1750-1914 », Documents pour l'histoire des techniques [En ligne], 18 | $2^{\mathrm{e}}$ semestre 2009, mis en ligne le 24 septembre 2010, consulté le 10 décembre 2020. URL : http://journals.openedition.org/dht/285 ; DOI : https://doi.org/10.4000/dht.285

Ce document a été généré automatiquement le 10 décembre 2020.

(c) Tous droits réservés 


\section{Christine Macleod, Heroes of} invention. Technology, liberalism and British identity 1750-1914

Cambridge, Cambridge university press, 2007, 458 pages.

Liliane Pérez

\section{RÉFÉRENCE}

Christine Macleod, Heroes of invention. Technology, liberalism and British identity 1750-1914, Cambridge, Cambridge university press, 2007, 458 pages. 
1 Deux courants de recherche ont contribué, ces dernières années, à redessiner l'histoire de la première révolution industrielle. D'une part, les données empiriques ont continué d'être revues au prisme d'une approche multifactorielle de la croissance, différenciée à l'échelle locale et connectée à l'échelle du monde. D'autre part, la notion de "révolution industrielle » est devenue en soi un objet d'histoire, comme mythe fondateur de l'identité nationale britannique («Britishness») depuis le $\mathrm{XIX}^{\mathrm{e}}$ siècle.

Le livre de Christine MacLeod s'inscrit dans ce deuxième registre d'analyse. Il participe des débats importants nés dans le sillage de l'étude de David Cannadine pour qui l'introduction du concept de

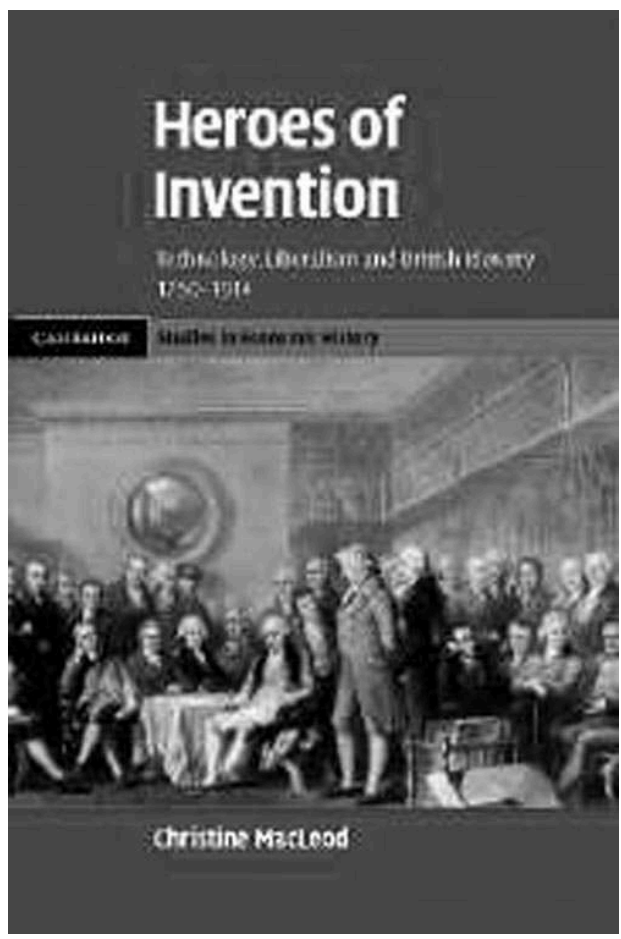
« révolution industrielle » en GrandeBretagne dans les années 1880 (notamment par Arnold Toynbee) était liée à une vision catastrophiste du changement économique, à mesure que le coût social de l'industrialisation était critiqué ${ }^{1}$. La conscience de l'impact de l'industrialisation sur les populations et sur l'environnement avait grandi depuis le milieu du siècle et avait contribué à acclimater l'idée, née sur le Continent en même temps que l'expression de "révolution industrielle ", d'une transformation radicale de la production. À la suite de Cannadine, Gareth Stedman Jones, tout en confortant ce point de vue, a mis en valeur le rôle de la célébration Whig de l'industrie dans les années 1880 (y compris chez Toynbee), tant l'essor industriel permettait d'écarter tout changement social violent sur le mode de la Révolution française et d'effacer la mémoire du combat républicain en Grande-Bretagne ${ }^{2}$. Au pire, la « révolution industrielle » était associée aux griefs contre le progrès, montant depuis les années 1850 ; au mieux, elle venait à occuper (non sans mal) une fonction dans le récit historique britannique à la fin du $\mathrm{XIX}^{\mathrm{e}}$ siècle au sein d'une vision libérale de la croissance.

3 C. MacLeod propose une troisième voie. Dans le chapitre introductif, elle rejette vivement les arguments qui ont contribué à minimiser le poids politique et culturel de la révolution industrielle avant 1880 , y compris le discours sur le "déclin industriel britannique " qui s'est déployé au $\mathrm{XX}^{\mathrm{e}}$ siècle, instillant une légende noire des inventeurs à partir du milieu du XIX siècle. Au contraire, l'auteure explique que la célébration des inventeurs a suscité un intérêt croissant, de même que la notion de " révolution industrielle ", à partir des années 1820-1830, quand un monument à la gloire de James Watt fut érigé dans Westminster (1834). Ce mouvement culmina dans le troisième quart du XIX ${ }^{\mathrm{e}}$ siècle, à la suite de l'exposition du Crystal Palace (1851). L'ère victorienne fut l'âge des inventeurs. Faisant écho à l'interprétation de l'identité nationale par Linda Colley, C. MacLeod montre que la société civile, et non les élites politiques ou le gouvernement, a forgé ce culte de l'inventeur. Dans les années 1850, un 
faisceau de facteurs a conduit à l'avènement de l'inventeur comme symbole nouveau de la « Britishness».

4 Certains traits de ces changements dans la société et la culture couraient sur le long terme. Dans le chapitre deux, l'auteur rappelle les images complexes et souvent négatives des inventeurs depuis la Renaissance et leur reconnaissance graduelle au siècle des Lumières, à mesure que le providentialisme de l'invention est remis en cause au profit de la créativité et de l'ingéniosité humaines. Le chapitre suivant est dévolu à l'intérêt pour l'invention technique au XVIII e siècle en Grande-Bretagne. Les sites industriels prennent place dans le Grand Tour; des savants, des hommes d'État et quelques éditeurs radicaux commencent à opposer aux gloires militaires des héros pacifiques, promouvant Newton, Brindley, Smeaton comme des «bienfaiteurs nationaux ». Si la réputation des inventeurs outre-Manche n'égale pas le culte civique développé en France à l'époque, leur renommée s'accroît et les récompenses votées par le Parlement ne sont pas seules en cause. En 1823, James Watt, et non Edward Jenner (pourtant primé par le Parlement) est glorifié et la machine à vapeur célébrée dans le premier numéro du Mechanic's Magazine, reprenant en l'adaptant le frontispice de Francis Bacon's pour l'Instauratio magna.

$5 \quad$ Les chapitres quatre et cinq présentent le rôle crucial joué par le culte de James Watt développé après sa mort (1819). La mémoire de Watt est née d'un combat politique entre les réformateurs (Whigs et Tories libéraux) et le pouvoir aristocratique et militaire, fortement consolidé après vingt ans de guerre contre la France. Lors de la réunion extraordinaire tenue à Westminster en juin 1824, présidée par Lord Liverpool (Tory libéral) et rassemblant des " hommes de science, de lettres et de commerce », une souscription est lancée pour un monument commémoratif dans l'abbaye de Westminster. William Huskisson, président du Board of Trade et avocat du libreéchange, glorifie la vapeur comme nouvelle source de domination britannique en temps de paix et d'extension de la civilisation chrétienne à travers le monde. Mais Londres n'est pas encore prêt à mener ce combat. C. MacLeod décrit le triomphe de Watt en Écosse où des monuments sont élevés à partir de 1824 (à Greenock et Glasgow), où les "intellectuels Whig", les entrepreneurs de coton et une large gamme de métiers, y compris des ouvriers qualifiés, partagent leur admiration pour Watt comme « mécanicien ». À Manchester, les manufacturiers Tory soutiennent les causes libérales, aux côtés des institutions locales et une statue est érigée en 1857. L'auteure souligne la relation entre la renommée croissante de Watt et le Reform Act de 1832 qui promeut les classes moyennes dans la vie civique. Bien que des résistances se fassent jour et que l'amiral Nelson soit encensé par les élites aristocratiques, l'apothéose de Watt inaugure la « révolution industrielle ». Le constat de C. MacLeod est crucial. À partir des années 1830 , la machine à vapeur "est vue comme le principal agent historique ", pour le meilleur (selon les libéraux et partisans du libre-échange) ou pour le pire: Peter Gaskell, en 1833, voit dans la vapeur une "révolution complète" aux effets dévastateurs. Avec l'apologie de la vapeur, «une nouvelle histoire de la GrandeBretagne est ratifiée ». La notion de "révolution industrielle (à défaut du terme précis) est fermement établie durant les décennies médianes du XIX ${ }^{e}$ siècle ». Une telle célébration de la technique a tendu à être oubliée quand la "révolution industrielle " est entrée dans le monde académique, à la fin du siècle, en même temps que s'affirmaient les vues pessimistes de l'industrialisation. 
6 De plus, le triomphe de Watt soulève de nouvelles questions dévolues à l'analyse du "génie inventif», décrit comme la puissance du pouvoir analogique, source de la supériorité des "grands inventeurs ", alors que les radicaux, comme Thomas Hodsgkin, prétendent que l'activité inventive est un effort collectif. Si les radicaux se joignent tardivement et avec ambiguïté au culte de Watt forgé par leurs ennemis politiques, Hodgskin fournit la première dénégation au malthusianisme: non sans écho à la rhétorique providentialiste, il affirme que la croissance de la population favorise l'amélioration du niveau de vie parce que le capital de la nation, c'est «le savoir-faire des ouvriers" (comme Watt) qui, collectivement sont à l'origine de la division du travail. D'autres auteurs radicaux conjuguent l'éloge de la réussite collective et de l'effort individuel, alors que le soutien au droit de l'inventeur devient une question d'intérêt majeur dans les années 1850.

7 Avant d'en venir à cet important sujet, C. MacLeod fournit, au chapitre sept, l'étude détaillée de la fabrique du "Panthéon technique » et du processus de sélection qui a favorisé la mémoire des Stephenson et des Brunel, au contraire de Richard Arkwright, vu comme un entrepreneur, non comme un génie technicien. Au milieu du siècle, le culte des ingénieurs est promu par les associations professionnelles, par les auteurs populaires radicaux comme Samuel Smiles, par la presse, à l'échelle locale et nationale. La nouveauté spectaculaire des chemins de fer favorise un consensus qui rallie les élites aristocratiques au culte du progrès. Cette évolution dérive de trois facteurs principaux : le succès du libre-échange, la défense du système des brevets (patents) et l'implication de la classe ouvrière dans le culte rendu aux inventeurs.

En premier lieu intervient la défense du libre-échange et ses liens de plus en plus étroits la cause des inventions techniques, comme l'illustre le Crystal Palace. Au chapitre huit, l'auteure explique que les inventeurs deviennent des « héros de la Pax Britannica » et sont honorés comme tels par l'État. La vapeur et le télégraphe sont particulièrement prisés comme des moyens de "résolution pacifique des conflits ». Le tableau The distinguished men of science (1862) figurant sur la couverture du livre, est emblématique du succès des inventeurs, considérés comme les égaux des savants. C'est une victoire fragile ; la Guerre de Crimée revigore les héros militaires et les inventeurs perfectionnant l'armement, comme Lord Armstrong, Bessemer, Siemens, anoblis à la fin du siècle, comme tous les grands ingénieurs civils.

Mais, en deuxième lieu, le triomphe des inventeurs excède largement les honneurs rendus par l'État. Dans le chapitre neuf, C. MacLeod, spécialiste de l'histoire des patents, montre que la controverse sur les droits de propriété n'améliore pas seulement la situation juridique (et financière) des inventeurs mais accroît leur renommée grâce à la réforme de 1852. Bennett Woodcroft, en charge du tout nouveau Patent office, joue un rôle essentiel. Il commence à collecter et à imprimer les patents et emplit le Patent office museum, créé dans le sillage du Crystal Palace, de portraits d'inventeurs et modèles d'inventions, telle la Rocket de Stephenson. À la suite, les biographies d'inventeurs foisonnent dans les années 1860. Si les débats sont vifs à propos du système des patents, notamment car s'expriment des arguments collectivistes et déterministes forts (dont ceux de Marx) plaidant pour l'abolition des droits exclusifs, la valorisation de l'initiative personnelle (" self-help») gagne la bataille. Les économistes, dès lors, se détournent de l'invention et du déterminisme pour se concentrer sur les impacts destructifs du machinisme. C'est aux États-Unis qu'est appelée à se développer l'économie de l'invention. 
10 La troisième composante du triomphe des inventeurs tient à leur image comme représentants de «l'aristocratie du travail ». À nouveau, l'agenda politique, le Reform Act de 1867, renforce la légitimité des inventeurs. Les " grands inventeurs » servent la cause des artisans en quête de respectabilité. Le soutien vient de Samuel Smiles qui met au défi la gloire de la «naissance noble » face au mérite (« self-improvement») et au caractère. Les syndicats, sur leurs certificats et leurs bannières, honorent les grands inventeurs et se font les avocats de la paix et du progrès. La classe ouvrière et les instituts de génie mécanique sont les principaux artisans de la «manie» des statues (un média visuel important à l'époque). Ils revendiquent ainsi la «reconnaissance de leur propre contribution à la prospérité du pays ». La mémoire de l'invention a été un combat et dans certains cas, les ouvriers ont été les promoteurs du culte de l'inventeur, comme pour Samuel Crompton et Humphry Davy.

11 Dans les deux derniers chapitres, C. MacLeod analyse l'effacement de la gloire des inventeurs à la fin du XIX ${ }^{e}$ siècle : le libre-échange ne suscite plus de débat, les patents sont à nouveau réformés en 1883 et la franchise parlementaire est acquise pour les ouvriers qualifiés depuis 1867. De plus, la renommée des inventeurs est remise en cause par celle des ingénieurs, des entrepreneurs et des savants, jouissant tous d'une identité professionnelle et d'un statut d'élite comme cela devient évident à la mort de Siemens en 1883. Le chapitre onze est consacré à la montée en puissance des ingénieurs et des entrepreneurs dans le Panthéon national. L'Institution of Civil Engineers joue un role clé depuis les débuts. C. MacLeod, après avoir étudié la statuaire des inventeurs, adopte ici un point de vue original et particulièrement pertinent en analysant les vitraux de l'abbaye de Westminster dévolus aux ingénieurs ("the railway triumvirat»). Loin de tout " déclin de l'esprit industriel ", les vitraux célèbrent les succès des ingénieurs et les intègrent à l'élite de la nation. Autre figure de l'élite bourgeoise, l'entrepreneur, encensé par Joseph Schumpeter en 1912 comme le principal agent de l'innovation, détrône l'inventeur indépendant (un changement dans lequel la classe ouvrière joue aussi un rôle comme le montre l'auteure avec finesse). Alors que les inventeurs commencent à être associés à de piteux hommes d'affaires, ils sont seulement sauvés par l'histoire locale et la popularité des héros régionaux, surtout en Écosse et en Cornouaille. L'assaut final est donné par les savants qui s'opposent avec véhémence à la gloire des inventeurs et revendiquent la supériorité de la science sur la technique. Si au milieu du siècle, les inventeurs étaient considérés par la British Association comme "des hommes de science ", les savants se plaignent maintenant du «déclin » de la science. Ils fustigent la négligence de la Royal Society et du gouvernement, alors que les inventeurs bénéficient des profits autorisés par les patents. À partir des années 1870, «la science appliquée » reçoit plus d'attention que l'invention. Le succès de l'image du savant-inventeur l'atteste, comme celle de Henry Perkin. L'invention technique suscite au mieux l'indifférence (comme une activité banale et automatique), au pire l'angoisse (à cause des usages militaires) ou l'antipathie à mesure que la face sombre de l'industrialisation donne corps à l'histoire de la révolution industrielle. Les inventeurs ne sont plus des héros lors de la deuxième révolution industrielle. Dans l'épilogue, l'auteure rappelle que cette évolution n'a en rien été inversée au XXe siècle, le culte du savant et la montée d'explications déterministes du changement technique ayant accentué le retrait des inventeurs indépendants.

12 Alors que certains historiens ont revu à la baisse la perception que les contemporains avaient de la révolution industrielle avant la fin du XIX ${ }^{e}$ siècle ou l'ont réduite au seul 
rejet du machinisme, alors que d'autres ont vu en ceci la racine du « déclin industriel britannique ", C. MacLeod établit de manière extrêmement convaincante que les Victoriens ont été pleinement conscients de leurs réalisations industrielles et qu'ils ont glorifié les inventeurs comme génies de la nation. Si le culte de l'inventeur s'efface dans les années 1880 , sa mémoire a rendu possible l'écriture d'une histoire économique britannique conçue comme une "révolution industrielle » et la diffusion d'un récit positiviste de l'invention au $\mathrm{XX}^{\mathrm{e}}$ siècle. En réhabilitant la culture industrielle comme composante majeure de l'identité nationale, non seulement $\mathrm{C}$. MacLeod fraie une voie pour relire et subvertir la mythologie du progrès héritée de cette période mais elle identifie le lien trop souvent oublié entre l'invention technique et l'émancipation politique. Ce livre, véritable chef d'œuvre, confirme que l'histoire des techniques est au cœur du renouvellement actuel des approches dans l'histoire intellectuelle et politique de la modernité.

\section{NOTES}

1. David Cannadine, «The past and present of the English Industrial Revolution 1880-1980", Past and present, 103, 1984, pp. 104-131.

2. Gareth Stedman Jones, La fin de la pauvreté ? Un débat historique (2004), Paris, Édition Ère, 2007.

\section{AUTEURS}

LILIANE PÉREZ

CDHTE-Cnam 